\title{
Association between Continuity of Care and Health-Related Quality of Life
}

\author{
Elizabeth A. Bayliss, MD, MSPH, Jennifer L. Ellis, MSPH, Jo Ann Shoup, PhD, MSW, \\ Deanna B. McQuillan, MA, John F. Steiner, MD, MPH, and Chan Zeng, PhD
}

Background: Patient-reported outcomes (PROs) are considered potential quality metrics for patients with multiple chronic medical conditions (MCC). Although continuity of care (COC) is an essential MCC care process, the association between common PROs and COC is unknown.

Methods: We assessed baseline and two-year follow-up self-reported health status, physical, and emotional well-being, and COC in seniors with MCC. Using mixed effects models with repeated measures adjusting for age, gender, and morbidity, we assessed each outcome as a function of COC.

Results: Of 2,078 seniors, 961 completed the initial survey and 806 completed follow-up. On a 0-100 scale, mean (sd) baseline self-reported health status, physical well-being, and emotional wellbeing were $48.7(22.0), 36.4(11.4)$, and 54.8 (9.0). On a 0 to 1 scale, mean baseline and 2 -year COC were 0.24 (sd 0.22$)$ and $0.22(0.18)$. Follow-up self-reported health status, physical well-being, and emotional well-being were 48.8 (23.1), 36.5 (11.5), and $55.3(8.8)$. In adjusted primary and secondary analyses using all available data, there were no associations between any outcomes and COC.

Conclusion: Given the measurement burden of quality assessment, negative associations between potential quality metrics and care processes are informative. Systematic assessment of PROs can inform patient-centered MCC care. However, PRO scores should be used with caution as quality measures. (J Am Board Fam Med 2017;30:205-212.)

Keywords: Chronic Disease, Comorbidity, Continuity of Care, Follow-up Studies, Health Status, Patient-Reported Outcome Measures, Quality of Life, Quality of Health Care, Self Report, Surveys and Questionnaires

Patient-reported outcome (PRO) measures such as self-reported health status and physical and emotional well-being are increasingly promoted as potential measures of care quality. ${ }^{1-7}$ Because PROs capture domains that cross conditions and reflect patient-centered priorities, such measures may more accurately reflect the health and well-being of individuals with multiple chronic medical condi-

This article was externally peer reviewed.

Submitted 13 July 2016; revised 25 October 2016; accepted 1 November 2016.

From the Institute for Health Research, Kaiser Permanente Colorado, Denver (EAB, JLE, JAS, DBM, JFS, CZ); the Department of Family Medicine, University of Colorado School of Medicine, Aurora (EAB); and the Department of Internal Medicine, University of Colorado School of Medicine, Aurora (JFS).

Funding: This investigation was funded by the Agency for Healthcare Research and Quality (1R01 HS018404-01).

Conflict of interest: none declared.

Disclaimer: The funder had no role in the study design; collection, analysis, and interpretation of data; writing of the report; or decision to submit the article for publication. tions (MCCs) than do common disease-specific quality measures. ${ }^{8,9}$ Across populations, higher scores on these and other domains of health-related quality of life (HRQOL) correlate with lower rates of hospitalization, lower overall mortality, lower cardiovascular morbidity, and lower outpatient services utilization in populations of patients with chronic illness. ${ }^{10-12}$

Although PROs may accurately capture the health of individuals with MCCs, promoting them as quality measures implies that there is evidence linking recommended care processes to these outcomes, and that measuring PROs can evaluate and inform the delivery of care. ${ }^{13,14}$ However, it is unclear whether recommended elements of care delivery for patients with MCCs affect the most com-

Corresponding author: Elizabeth A. Bayliss, MD, MSPH, Institute for Health Research, Kaiser Permanente, 10065 E. Harvard Ave., Suite 300, Denver, CO 80231-5968 (E-mail: elizabeth.bayliss@kp.org). 
monly measured PROs that capture domains of HRQOL. Health status, functional status, and emotional well-being are multifactorial constructs, and scores may or may not change based on receiving high- or low-quality care.

One care process that is essential to high-quality care for MCCs is optimal continuity of care (COC). For the population with MCCs, greater COC is associated with lower rates of hospitalization and emergency service use, better control of chronic diseases, greater patient satisfaction, greater patient trust and communication with one's physician, and lower mortality rates. ${ }^{15-18}$ Although it has been suggested that patient-reported HRQOL could reflect care processes specifically including COC, this specific association has not been evaluated. ${ }^{19}$

Given this gap in knowledge, we investigated the longitudinal association between COC and 3 domains of HRQOL: self-reported health status, functional status, and emotional well-being. We hypothesized that if there is an association between COC and HRQOL, greater COC would be associated with better HRQOL in a population of seniors with MCCs.

\section{Methods}

\section{Study Design and Setting}

We conducted a 2-year prospective telephone survey to assess HRQOL as a function of interpersonal COC among patients in Kaiser Permanente Colorado, a not-for-profit, integrated health care delivery system. The Institutional Review Board of Kaiser Permanente Colorado reviewed and approved the study.

\section{Study Population}

The study population consisted of 961 survey respondents from among a random sample of 2078 adult members of Kaiser Permanente Colorado who were aged $\geq 65$ years on January 1, 2010; had at least 1 year of enrollment before and at least 2 years of enrollment following this date; and had $\geq 3$ of 10 common chronic medical conditions. We excluded individuals with a diagnosis of dementia or who recently or currently required skilled nursing care. Respondents were, on average, 1 year younger than nonrespondents; the 2 groups did not differ with regard to diagnosis-based morbidity level or sex.

\section{Measures}

HRQOL was measured at baseline and 2 years later using the 36-item Rand questionnaire to assess selfreported health status, physical function, and emotional well-being. ${ }^{5} \mathrm{We}$ measured interpersonal COC using the COC Index (COCI) ${ }^{20}$ which captures the concentration of visits to individual providers and is sensitive to the number of providers seen-which is particularly relevant to individuals with multimorbidity. The COCI formula is as follows: COCI $=\sum_{j=1}^{s}{ }_{j} n_{\mathrm{j}}^{2}-N / N(N-1)$, where $\mathrm{N}$ is the total number of visits, $\mathrm{n}_{\mathrm{j}}$ is the number of visits to provider $\mathrm{j}$, and $s$ is the total number of providers seen. Calculating a stable COCI value requires $\geq 3$ visits. Therefore, for each outcome, we required $\geq 3$ outpatient visits to a primary and/or specialty care clinician during each measurement period. COC was measured during the year before baseline and during the 2-year period between baseline and follow-up. Thus each respondent had up to 2 measures of COC and HRQOL, with each COC measure temporally preceding each measure of HRQOL (Figure 1). Chronic conditions were identified by International Classification of Diseases, Ninth Revision codes listed in the electronic health record during the year before baseline.

\section{Analysis}

To assess the domains of HRQOL as a function of COC and time, we used a mixed effects model with a maximum likelihood estimation approach to analyze repeated HRQOL measures collected at baseline and follow-up, adjusting for age, sex, and morbidity burden. We also conducted secondary analyses using multiple imputation to account for missing data. Both maximum likelihood and multiple imputation methods assume data are missing at random, which allows the probability of missingness to depend on observed data and not on missing

Figure 1. Measurement timeline for health-related quality of life (HRQOL) domains and continuity of care (COC). T0, Time zero; Y1, year 1; Y2, year 2; Y3, year 3 .

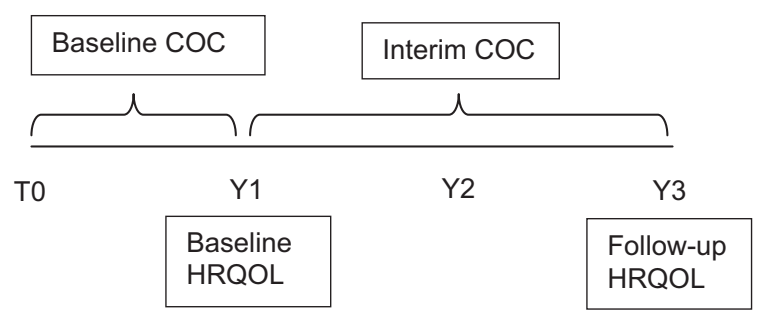


data. ${ }^{21,22}$ Given the number of predictors we considered in the regression model, the sample size in our study population provided $80 \%$ power to detect a small effect size $\left(R^{2}=0.02\right)$ at $\alpha=0.05 .^{23}$ This effect size is consistent with minimally clinically significant differences in HRQOL scores. ${ }^{24}$

\section{Results}

Of the 961 seniors who responded to the initial survey, 806 completed the 2-year follow-up. Figure 2 illustrates cohort development and Table 1 describes population characteristics. Losses to follow-up were primarily the result of death $(n=68)$; ineligibility because of dementia, skilled nursing care, or disenrollment $(\mathrm{n}=45)$; and refusal $(\mathrm{n}=$ 21). On a normed scale of 0 to 100 , mean (standard deviation) self-reported health status, physical wellbeing, and emotional well-being were 48.7 (22.0), 36.4 (11.4), and 54.8 (9.0), respectively, at baseline. Among cohort members with enough visits to calculate COCI, mean overall COCI was $0.24(0.22)$ on a 0 -to- 1 scale at baseline and $0.22(0.18)$ during the 2-year follow-up. Minimal change in HRQOL domains occurred over 2 years. Mean (standard deviation) self-reported health status, physical wellbeing, and emotional well-being during follow-up were $59.6(21.2), 36.5(11.5)$, and 55.3 (8.8), respectively.

In adjusted repeated measures analyses using all available data, there was no significant association between any of the HRQOL domains and COC (Table 2). Greater morbidity burden was negatively associated with self-reported health status and physical and emotional well-being. There was minimal change in calculated morbidity level from baseline to follow-up. Female sex and older age were also negatively associated with physical well-being.

Participants with missing follow-up data because of disenrollment or reasons other than death had baseline characteristics similar to those with complete follow-up. Participants with missing follow-up data because of death were older, had greater morbidity, and had worse health status at baseline than those with completed follow-up $(P<$ .001 for all). The lack of an association between COC and HRQOL did not change in secondary analyses using multiple imputation.

\section{Discussion}

In a health care delivery culture of substantial primary care measurement burden and misguided quality incentives, negative associations between

Figure 2. Consort diagram.

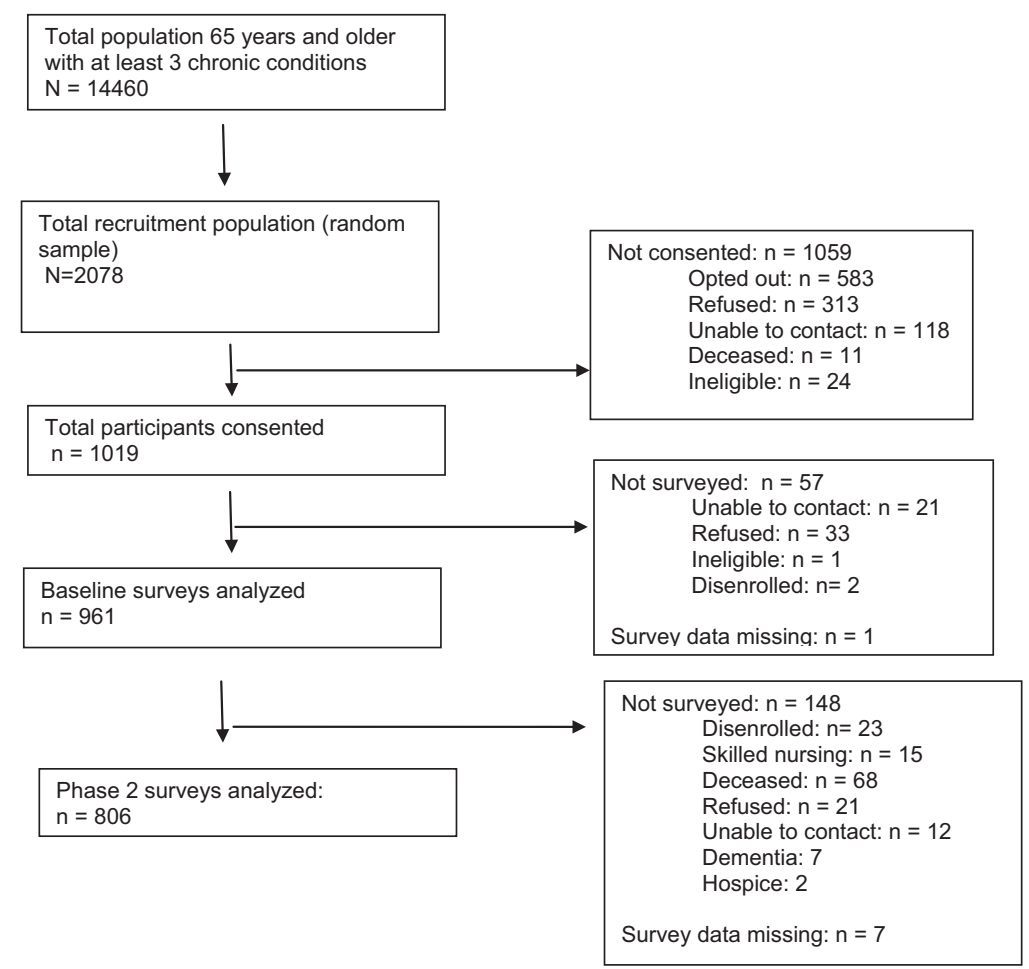


Table 1. Demographic, Clinical, and Outcome Characteristics of Seniors with Multiple Chronic Conditions at Baseline (2010) $(\mathrm{N}=961)$

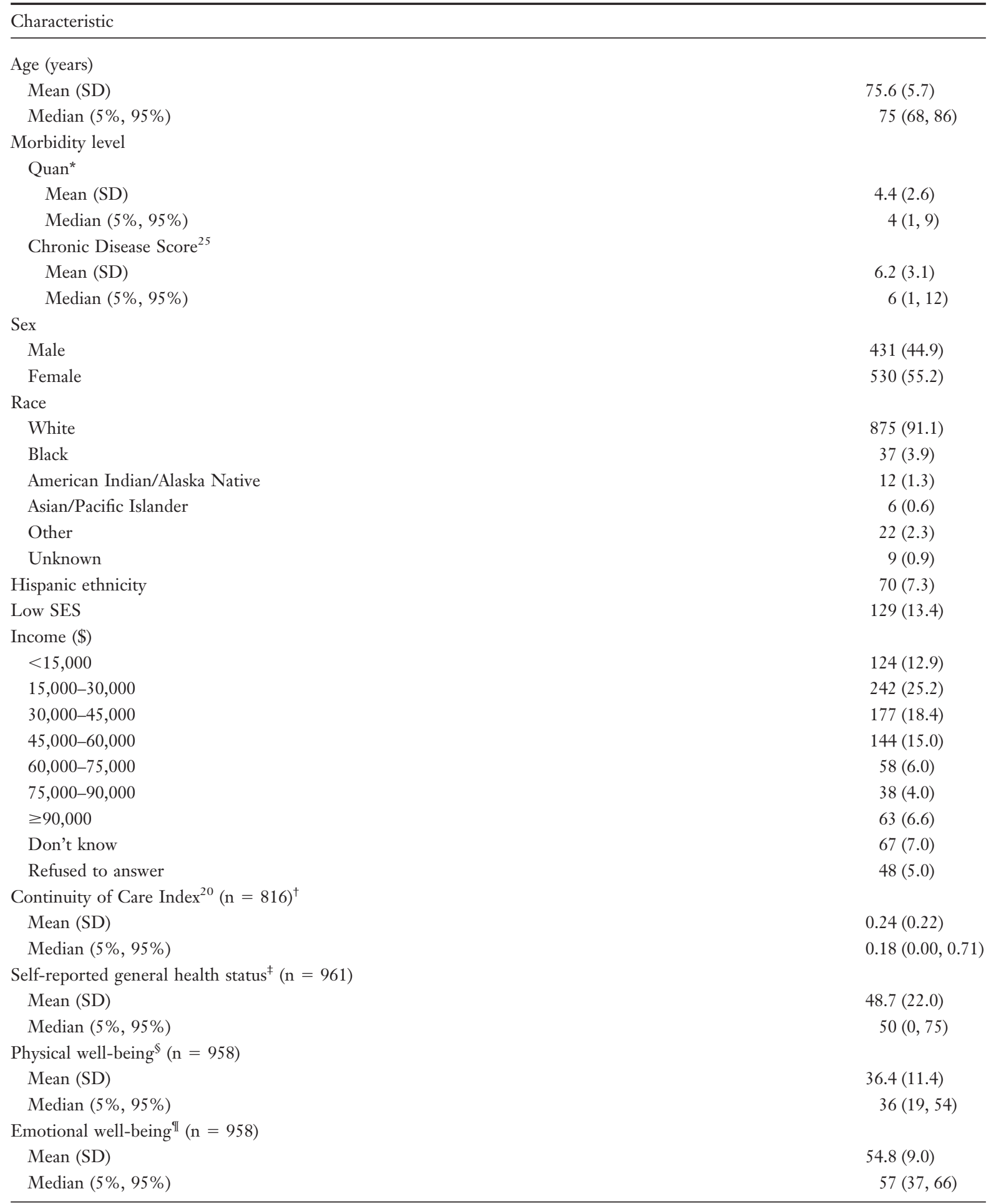

Data are number (\%) of participants unless otherwise indicated. Population norms for health-related quality of life measures $=50$. ${ }^{*}$ Quan adaptation of the Elixhauser Comorbidity Index. ${ }^{26}$

${ }^{\dagger}$ Primary and specialty care, year before baseline.

${ }^{\ddagger}$ Rated as excellent, very good, good, fair, or poor.

${ }^{\S}$ Score from the Physical Component Summary of the 36-item Rand instrument.

"Score from the Mental Component Summary of the 36-item Rand instrument.

SD, standard deviation; SES, socioeconomic status. 
Table 2. Adjusted Associations Between HealthRelated Quality of Life and Continuity of Care

\begin{tabular}{|c|c|c|c|}
\hline Outcome & Estimate & $\begin{array}{c}\text { Standard } \\
\text { Error }\end{array}$ & $P$ Value \\
\hline \multicolumn{4}{|c|}{ Self-reported health status* } \\
\hline $\mathrm{COC}$ & -1.0140 & 2.4976 & .6848 \\
\hline Time & -0.6441 & 0.7656 & .4005 \\
\hline Age & -0.1461 & 0.1180 & .2158 \\
\hline Female sex & -2.4423 & 1.3275 & .0661 \\
\hline Quan $\mathrm{ECI}^{25}$ & -2.4220 & 0.2593 & $<.0001$ \\
\hline \multicolumn{4}{|c|}{ Physical well-being $^{\dagger}$} \\
\hline $\mathrm{COC}$ & -0.0886 & 1.1783 & .9401 \\
\hline Time & -0.0166 & 0.3455 & .9616 \\
\hline Age & -0.2626 & 0.0595 & $<.0001$ \\
\hline Female sex & -2.6292 & 0.6705 & $<.0001$ \\
\hline Quan $\mathrm{ECI}^{25}$ & -1.0025 & 0.1308 & $<.0001$ \\
\hline \multicolumn{4}{|c|}{ Emotional well-being $^{\ddagger}$} \\
\hline $\mathrm{COC}$ & 0.6339 & 1.0623 & .5508 \\
\hline Time & 0.4004 & 0.3309 & .2266 \\
\hline Age & -0.0205 & 0.0477 & .6666 \\
\hline Female sex & -0.7597 & 0.5352 & .1561 \\
\hline Quan $\mathrm{ECI}^{25}$ & -0.3495 & 0.1046 & .0009 \\
\hline
\end{tabular}

Bold indicates associations significant at $P<=.05$.

*Rated as excellent, very good, good, fair, or poor by 961 patients (1602 observations).

${ }^{+}$Score from the Physical Component Summary of the 36-item Rand instrument (completed by 961 patients [1598 observations]).

${ }^{\ddagger}$ Score from the Mental Component Summary of the 36-item Rand instrument (completed by 961 patients [1598 observations]).

COC, continuity of care; Quan ECI, Quan adaptation of the Elixhauser Comorbidity Index.

potential quality metrics and important care processes are informative. PROs have been promoted as a means to evaluate the effect of clinical processes and interventions on elements of health and well-being. ${ }^{27}$ Based on this analysis, self-reported health status and physical and emotional well-being do not seem to be associated with COC. This suggests that these well-validated and easily quantifiable PROs may not accurately reflect the important processes of care for MCCs and should be used with caution as a broad quality measure for this population.

National Quality Forum criteria for developing quality measures from PROs specifies that they be psychometrically sound, person-centered, meaningful, and amenable to change. ${ }^{2}$ Importantly, they must also be valid and responsive to the care process or intervention. ${ }^{2,28}$ This has been demonstrated for physical and emotional well-being scores in response to specific interventions such as joint replacement and depression treatment. ${ }^{29,30} \mathrm{In}$ our analysis, health status and physical and emotional well-being as functions of COC did not meet these criteria. In the context of primary care delivery especially, care processes and outcomes are multifactorial (Figure 3). As an outcome, HRQOL is a broad construct that reflects biological factors, functional status, social and emotional well-being, environmental influences, expectations of health, and disease-specific symptoms. ${ }^{31-34}$ Although HRQOL is associated with morbidity burden as measured by diagnoses, diagnosed conditions account for only part of overall HRQOL. ${ }^{35}$ As a care process, COC is only 1 aspect of patient-centered care that may affect health outcomes, and even optimal chronic disease care may not markedly affect overall morbidity burden-especially in the short term. ${ }^{34}$ Generating evidence to promote optimal care for individuals with MCCs requires an understanding of the multiple and complex associations between these processes and outcomes.

When asked, patients with MCCs describe high-quality care as being empathic and respectful, thorough, continuous, efficient, safe, and of high technical quality. ${ }^{35-39}$ Systematically assessing patient-reported information as part of an annual health assessment can guide patient-centered care and shared decision making. ${ }^{40}$ When PROs have been systematically integrated into care delivery, patients find the ensuing discussions helpful and patient-centered. ${ }^{41}$ However, assessing and acting on patient-reported information may not change individual or population-level scores of the measures themselves. ${ }^{27,34,42,43}$ Thus it may be the process of PRO assessment and clinical action that reflects high-quality care and not necessarily the change in PRO score. This interpretation of PRO use is consistent with calls for "reimagining" quality assessment to focus on measuring important processes, with less emphasis on outcomes. $^{44}$

Our study has several limitations. All methods for managing missing data have inherent limitations. Some of our HRQOL data were missing because of death or functional decline, and some COC data were missing because of less contact with the system among individuals with lower morbidity. We followed literature-based recommendations for managing missing data under general missing-at-random conditions, but other methods 
Figure 3. Example processes and outcomes relevant to the care of individuals with multiple chronic conditions.

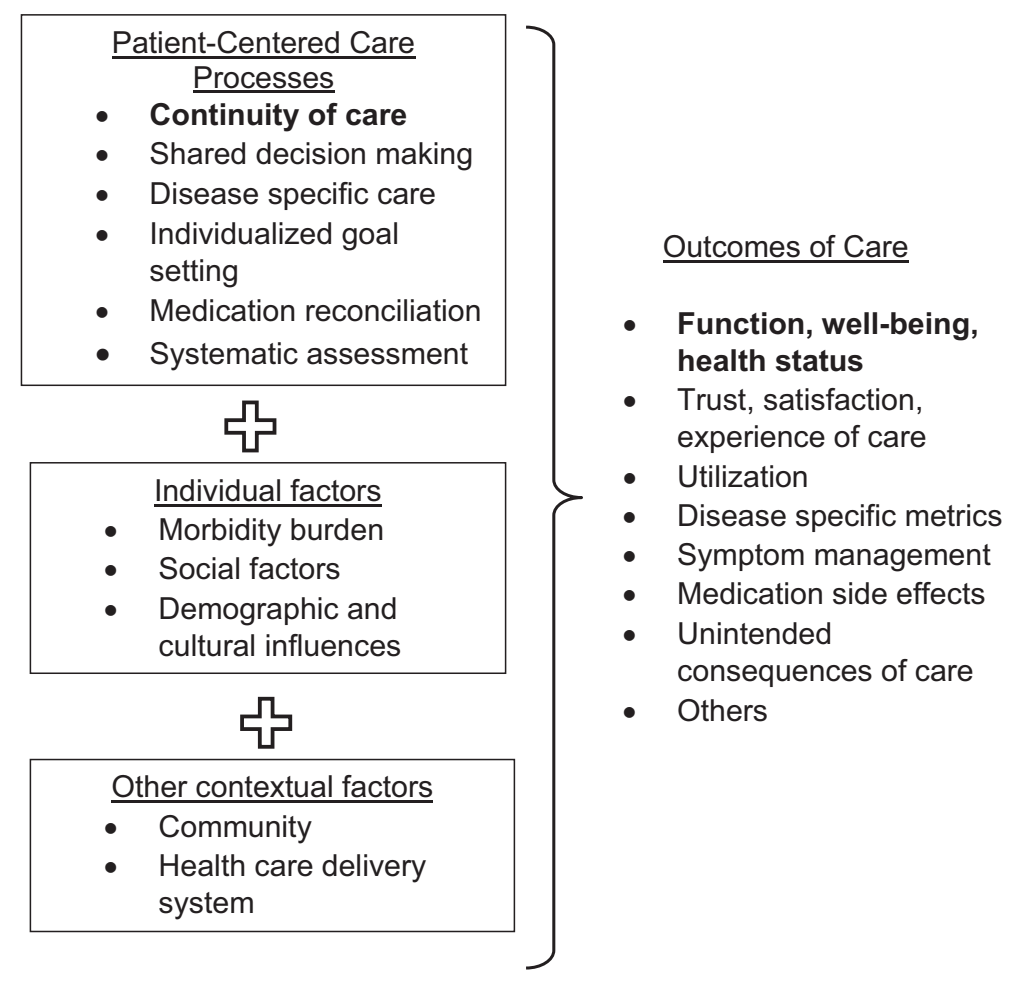

such as assigning zero values to HRQOL variables for known decedents have also been proposed. ${ }^{21,45}$ Based on primary and secondary analyses, we think it is unlikely that we missed a significant association between COC and HRQOL. Because our study was conducted in an integrated delivery system with a common electronic health record, our COC scores reflect relational continuity of care within that system; however, interpersonal COC remains relevant and improves outcomes within integrated systems. ${ }^{15}$ Our participants' mean COC score was in the low-to-medium range for geriatric populations. ${ }^{17,46}$ This likely does not reflect underascertainment, as claims data supplements electronic health record data to capture all primary and specialty care. Associations between HROQL and COC may differ in nonintegrated delivery settings or for populations with different distributions of COC and HRQOL. Finally, we only assessed the relationship between 3 domains of HRQOL and 1 important process of care. Further research is required to understand the multidimensional relationships between PROs and other care processes to adequately inform the use of PROs as a quality indicator for complex patient care.

\section{Conclusions}

Our findings illustrate the importance of selecting appropriate measures to evaluate and promote patient-centered care-especially for complex patient populations who benefit from multidimensional approaches to care delivery. Although systematic assessment of PROs can and should inform optimal care, assessing and acting on PROs may be more reflective of high-quality care for MCCs than the specific scores themselves, which may not reflect important care processes such as high COC.

To see this article online, please go to: bttp://jabfm.org/content/ 30/2/205.full.

\section{References}

1. Jensen RE, Rothrock NE, DeWitt EM, et al. The role of technical advances in the adoption and integration of patient-reported outcomes in clinical care. Med Care 2015;53:153-9.

2. National Quality Forum. Patient-reported outcomes in performance measurement. Washington, DC: National Quality Forum; 2013.

3. Van Der Wees PJ, Nijhuis-Van Der Sanden MW, Ayanian JZ, Black N, Westert GP, Schneider EC. Integrating the use of patient-reported outcomes for both clinical practice and performance measure- 
ment: views of experts from 3 countries. Milbank Q 2014;92:754-75.

4. Boyce MB, Browne JP, Greenhalgh J. The experiences of professionals with using information from patient-reported outcome measures to improve the quality of healthcare: a systematic review of qualitative research. BMJ Qual Saf 2014;23:508-18.

5. Hays RD, Sherbourne CD, Mazel RM. The Rand 36-item health survey 1.0. Health Econ 1993;2:217-27.

6. Bishop TF. Pushing the outpatient quality envelope. JAMA 2013;309:1353-4.

7. Alper J, Sanders J, Saunders R. Core measurement needs for better care, better health, and lower costs: counting what counts: workshop summary (2013). Washington, DC: National Academies Press; 2013.

8. Working Group on Health Outcomes for Older Persons with Multiple Chronic Conditions. Universal health outcome measures for older persons with multiple chronic conditions. J Am Geriatr Soc 2012; 60:2333-41.

9. Tinetti ME, McAvay GJ, Chang SS, et al. Contribution of multiple chronic conditions to universal health outcomes. J Am Geriatr Soc 2011;59:1686-91.

10. Fan VS, Au D, Heagerty P, Deyo RA, McDonell $\mathrm{MB}$, Fihn SD. Validation of case-mix measures derived from self-reports of diagnoses and health. J Clin Epidemiol 2002;55:371-80.

11. DeSalvo KB, Fan VS, McDonell MB, Fihn SD. Predicting mortality and healthcare utilization with a single question. Health Serv Res 2005;40:1234-46.

12. Miilunpalo S, Vuori I, Oja P, Pasanen M, Urponen H. Self-rated health status as a health measure: the predictive value of self-reported health status on the use of physician services and on mortality in the working-age population. J Clin Epidemiol 1997;50:517-28.

13. Brook R, McGlynn E, Cleary P. Quality of health care. Part 2: measuring quality of health care. N Engl J Med 1996;335:966-70.

14. McGlynn EA. Selecting common measures of quality and system performance. Med Care 2003;41(1 Suppl):I39-47.

15. Bayliss EA, Ellis JL, Shoup JA, Zeng C, McQuillan DB, Steiner JF. Effect of continuity of care on hospital utilization for seniors with multiple medical conditions in an integrated health care system. Ann Fam Med 2015;13:123-9.

16. Waibel S, Henao D, Aller M-B, Vargas I, Vázquez M-L. What do we know about patients' perceptions of continuity of care? A meta-synthesis of qualitative studies. Int J Qual Health Care 2012;24:39-48.

17. Nyweide DJ, Anthony DL, Bynum JP, et al. Continuity of care and the risk of preventable hospitalization in older adults. JAMA Intern Med 2013;173: $1879-85$.

18. Leleu H, Minvielle E. Relationship between longitudinal continuity of primary care and likelihood of death: analysis of national insurance data. PLOSE ONE 2013;8:e71669.

19. Tarlov AR, Ware JE Jr, Greenfield S, Nelson EC, Perrin E, Zubkoff M. The Medical Outcomes Study. An application of methods for monitoring the results of medical care. JAMA 1989;262:925-30.

20. Bice TW, Boxerman SB. A quantitative measure of continuity of care. Med Care 1977;15:347-9.

21. Schafer JL, Graham JW. Missing data: our view of the state of the art. Psychol Methods 2002;7:147-77.

22. Rubin DB. Inference and missing data. Biometrika 1976;63:581-92.

23. Cohen J. A power primer. Psychol Bull 1992;112: 155-9.

24. Hays RD, Morales LS. The RAND-36 measure of health-related quality of life. Ann Med 2001;33: 350-7.

25. Clark DO, Von Korff M, Saunders K, Baluch WM, Simon GE. A chronic disease score with empirically derived weights. Med Care 1995;33:783-95.

26. Quan H, Sundararajan V, Halfon P, et al. Coding algorithms for defining comorbidities in ICD-9-CM and ICD-10 administrative data. Med Care 2005;43: 1130-9.

27. Hostetter M, Klein S. Using patient-reported outcomes to improve health care quality. Quality Matters newsletter, December 2011/January 2012. New York: Commonwealth Fund; 2012.

28. Deutsch A, Smith L, Gage B, Kelleher C, Garfinkel D. Patient-Reported outcomes in performance measurement. Prepared by RTI International and Brookings Institution. Washington, DC:NQF; 2012. Available at http://www.qualityforum.org/Projects/ n-r/Patient-Reported_Outcomes/Patient-Reported_ Outcomes.aspx\#t $=2 \& s=\& p=2 \% 7 C$.

29. MacDonald SJ, Charron KD, Bourne RB, Naudie DD, McCalden RW, Rorabeck CH. The John Insall Award: gender-specific total knee replacement: prospectively collected clinical outcomes. Clin Orthop Relat Res 2008;466:2612-6.

30. Löwe B, Kroenke K, Herzog W, Gräfe K. Measuring depression outcome with a brief self-report instrument: sensitivity to change of the Patient Health Questionnaire (PHQ-9). J Affect Disord 2004;81: 61-6.

31. Wilson IB, Cleary PD. Linking clinical variables with health-related quality of life: a conceptual model of patient outcomes. JAMA 1995;273:59-65.

32. Garratt AM, Ruta DA, Abdalla MI, Russell IT. SF 36 health survey questionnaire: II. Responsiveness to changes in health status in four common clinical conditions. Qual Health Care 1994;3:186-92.

33. Revicki D, Hays RD, Cella D, Sloan J. Recommended methods for determining responsiveness and minimally important differences for patient-reported outcomes. J Clin Epidemiol 2008;61:102-9.

34. Kaplan SH, Kravitz RL, Greenfield S. A critique of current uses of health status for the assessment of 
treatment effectiveness and quality of care. Med Care 2000;38(9 Suppl):II184-91.

35. Bayliss EA, Ellis JL, Shoup JA, Zeng C, McQuillan DB, Steiner JF. Association of patient-centered outcomes with patient-reported and ICD-9-based morbidity measures. Ann Fam Med 2012;10:126-33.

36. Jenkinson C, Coulter A, Bruster S, Richards N, Chandola T. Patients' experiences and satisfaction with health care: results of a questionnaire study of specific aspects of care. Qual Saf Health Care 2002; 11:335-9.

37. Bendapudi NM, Berry LL, Frey KA, Parish JT, Rayburn WL. Patients' perspectives on ideal physician behaviors. Mayo Clin Proc 2006;81:338-44.

38. Sofaer S, Firminger K. Patient perceptions of the quality of health services. Annu Rev Public Health 2005;26:513-59.

39. Bayliss EA, McQuillan DB, Ellis JL, et al. Using electronic health record data to measure care quality for individuals with multiple chronic medical conditions. J Am Geriatr Soc 2016;64:1839-44.

40. Centers for Medicare and Medicaid Services. The ABCs of the annual wellness visit (AVW). Washington, DC: Department of Health and Human Services; 2015. Available from: https://www.cms.gov/ Outreach-and-Education/Medicare-LearningNetwork-MLN/MLNProducts/downloads/AWV_ chart_ICN905706.pdf. Accessed August 26, 2015.
41. Stover A, Irwin DE, Chen RC, et al. Integrating patient-reported outcome measures into routine cancer care: cancer patients' and clinicians' perceptions of acceptability and value. EGEMS (Wash DC) 2015;3:1169.

42. Kotronoulas G, Kearney N, Maguire R, et al. What is the value of the routine use of patient-reported outcome measures toward improvement of patient outcomes, processes of care, and health service outcomes in cancer care? A systematic review of controlled trials. J Clin Oncol 2014:32:1480-501.

43. Chung S, Lesser LI, Lauderdale DS, Johns NE, Palaniappan LP, Luft HS. Medicare annual preventive care visits: use increased among fee-for-service patients, but many do not participate. Health Aff (Millwood) 2015;34:11-20.

44. McGlynn EA, Schneider EC, Kerr EA. Reimagining quality measurement. N Engl J Med 2014;371: 2150-3.

45. Diehr P, Patrick D, Hedrick S, et al. Including deaths when measuring health status over time. Med Care 1995:33(4 Suppl):AS164-72.

46. Amjad H, Roth DL, Samus QM, Yasar S, Wolff JL. Potentially unsafe activities and living conditions of older adults with dementia. J Am Geriatr Soc 2016; 64:1223-32. 